\title{
DESIGN AND MYOELECTRIC CONTROL OF AN ACTIVE ORTHOSIS DEVICE USING FINITE STATE MACHINE ALGORITHM
}

\author{
Osman Ulkir ${ }^{1}$, Gazi Akgun ${ }^{2}$, Ersin Toptas ${ }^{3}$, Erkan Kaplanoglu ${ }^{4 *}$ \\ ${ }^{1}$ Department of Mechatronics Engineering, Mus Alparslan University, Mus, 49200, Turkey \\ ${ }^{2,3}$ Department of Mechatronics Engineering, Marmara University, Istanbul, 34730, Turkey \\ ${ }^{4 *}$ Department of Mechatronics Engineering, University of Tennessee at Chattanooga, TN, 37403, USA \\ e-mail: erkan-kaplanoglu@utc.edu
}

\begin{abstract}
This paper presents a novel myoelectric controlled active hip-knee-ankle-foot orthosis (A-HKAFO) designed to assist lower limb disorders. The proposed orthosis device2 consists of a polypropylene shell and a metal hinge joint, is designed to help patients during gait rehabilitation after neurological injury, and assist people who have difficulty walking3 due to obesity, sports injuries. The system also can use 4 for studying human gait biomechanics5. A myoelectric control law strategy is proposed using a finite state machine (FSM) method. The algorithm is activated by users' intend to forward or backward stepping6. The electromyogram (EMG) signals from lower limb7 and device motion data were8 used for the control of AHKAFO. In order to determine the last location of the user after movement, physical feedback is utilized from the mechanical system.
\end{abstract}

Keywords. Myoelectric control, orthosis device, finite state machine, HKAFO.

\section{INTRODUCTION}

In the world, about 80 million people have lower extremity disorders. Active orthotic devices are being developed for people who have diseased or weakened lower limb muscles. They have difficulty with walking due to trauma, brain stroke, spinal cord injuries, sports injuries, or obesity (Costa \& Darwin, 2006). There are two types of approaches to assistive systems for walking. The first is a Functional Electrical Stimulation (FES). In this approach, regaining muscle control of a patient's legs is provided by electrical stimulation (Hawran \& Fin, 1996). The other method is External Active Orthoses (EAO), where the support and power, necessary for the system, are provided by actuators (Lum et al., 2002).

Human walking is a combination of complex joint movements, and the identification of these movements has always been a challenge for medical specialists, engineers, and mathematicians. To provide this, a high degree of freedom of walking movement should be simplified. To overcome these problems, many researchers consider using biped robot models in their theoretical and experimental studies (Nagasaka et al., 1999). 
Regarding the design of active orthosis, there are some constraints such as the range of motion of the joints, kinematics of legs, the joint angle speed, and contact between links. To eliminate these limitations, it is necessary to perform a correct kinematical analysis of the system.

Generally, an active orthotic device expresses a device intended to increase the ambulatory capacity of a person suffering from leg pathology by providing some means of augmenting the power at one or more joints of the lower extremities. Thus, it helps to increase the power of the individual to support walking (Dollar \& Hugh., 2008). These systems are not suitable for people who have an absence of a muscular stimulus. Generally, the term 'exoskeleton' is used to describe certain rehabilitation devices, which includes the majority of the joints of the lower extremities.

These days, many wearable active orthosis devices have been successfully developed (Giovacchini et al., 2015; Tucker et al., 2015; Yan et al., 2015; Shorter et al., 2013; Ulkir et al., 2018;). The first known controllable active orthosis is a patent for a hydraulically actuated device from 1942 that added power at the hip and knee joints (Dollar \& Hugh., 2007). The MIT Biomechatronics Lab developed a powered ankle-foot orthosis to assist drop-foot gait, a deficit affecting many people who have suffered a stroke, suffer from multiple sclerosis, cerebral palsy, or another condition (Samuel et al., 2008). The device consists of a modified passive ankle-foot orthosis with the addition of a series elastic actuator. The active orthosis devices designed for such studies had electrical, hydraulic, and pneumatic actuators and different control methods (Sawicki et al., 2005; Ferris et al., 2006).

Muscle contraction in the human body gives rise to a bioelectrical signal proportional to the muscle activity. The signal is called Electromyogram (EMG), an important instrument for diagnosis, treatment, and the automatic control of the prosthesis and orthosis. Nowadays, orthotic systems use classical control techniques or EMG-based control.

This paper will look at the design of a portable device for the control of A-HKAFO, used for assisting lower limb disorders. With these kinds of devices, people will be able to have physical therapy and rehabilitation anytime, anywhere. During the design stage, a length-adjustable, motion-performable active orthotic device with three degrees of freedom (DoF) is utilized. This device includes rigid components that are divergent from a degree of freedom of human motion and can perform safety factors. The Brushed-DC motor is used for the actuation of the device and output of the improved EMGbased controller.

A control algorithm has been developed by using a finite state machine method, based on the acquired EMG signals and kinematics data taken from an accelerometer through the software.

This paper is constructed as follows. The first section describes the requirement of the size, kinematic design of the misalignment mechanism, and actuation unit of the A-HKAFO. Then control structure and EMG-based controller of the system are presented in the next section. The last section concludes this paper

\section{MATERIALS AND METHOD}

\section{Biomechanics of Walking}

Understanding the biomechanics of human walking is very crucial for the design of an active orthosis. Thus, it is necessary to explain the phases of human walking before describing the design. A simple scheme of the phases of human walking is shown in Figure 1 (Kanthi et al., 2014). During the loop of walking, the timing of events is taken approximately, as the changes are taken according to the person and conditions.

During walking, first, the person moves one of his/her legs to the front, removes the other from the ground, and moves forward when stepping with the first leg. In the walking cycle, the time where the leg stays in the air is called the "swing phase", and the time where the leg stays on the ground is the "stance phase." The moment of a lower extremity stepping on the ground is the beginning of the stance phase. When this extremity leaves the ground, the stance phase ends and the swing phase starts. When the same extremity steps into the ground again, the walking cycle is completed. 
In general, the human leg can be thought of as a seven DoF structure, with three rotational DoF at the hip, one at the knee, and three at the ankle. Human anatomical planes consist of three sections. These sections are the coronal, sagittal, and transverse planes.

The coronal plane, divides the body into the front and the rear sections, the sagittal plane which divides the body into the right and the left sections; and the transverse plane divides the body into upper and lower sections. The motion in the sagittal plane is known as "flexion" and "extension". The hip motion in the coronal plane is known as "abduction" and "adduction". The motion in the ankle in the coronal plane is called "eversion".

Figure 1: Human walking gait through one cycle
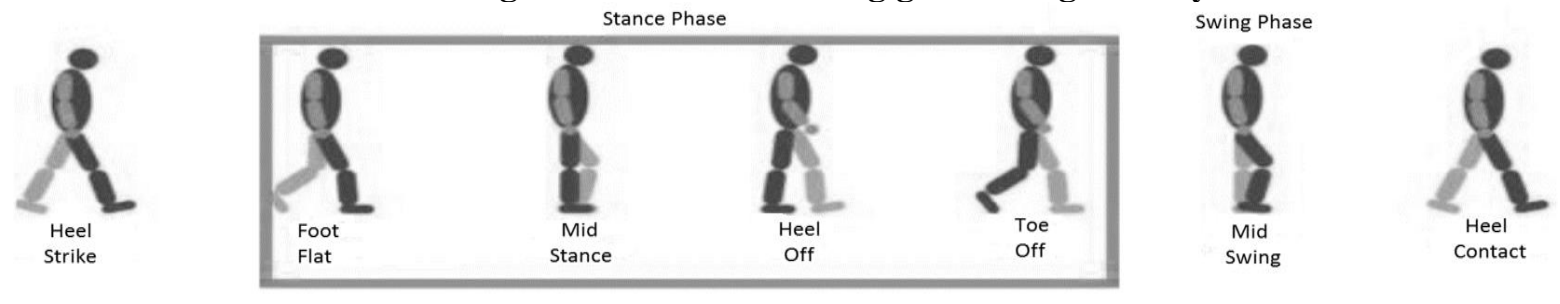

For our design, HKAFO is considered as a reference points. These orthoses are used to maintain the stabilization on the hip joint and control the rotation. In this study, with the help of an actuator system, located at the hip zone, an orthosis was put into action. These types of orthosis are called "active orthosis." Unlike passive orthosis, active orthoses have the potential to actively control the joints of the devices.

\section{Mechanical Structure of the HKAFO}

While the design of the orthosis device, the rules of biomechanics and suitability to the human anatomical structure should be considered. Another important criterion is the weights of the actuators and electrical equipment for easier carrying, and standing, of the device.

The 3D and rear view of the designed orthosis are shown in Figure 2. The orthosis is designed for people who are between 10-20 years old, have a maximum height of $170 \mathrm{~cm}$, and a maximum weight of $70 \mathrm{~kg}$. On the designed active orthosis model, the knee and ankle are considered rigid; but these parts can be activated anytime with lock mechanisms, which are located at the hip and knee joints. In this way, in consequence of any adverse motion, the limb can be prevented from incurring harm.

The A-HKAFO device consists of three sections: the hip, thigh, and calf. The material used in these sections is polyethylene, which is composed of polymers that member of the thermoplastics group. Polyethylene is a light and high strength material. It is a low density, slippery material with a low coefficient of friction. A fasteners and linkage mechanism consists of Al 6061 alloy. It has high corrosion resistance and excellent handling properties. It provides lightness in the orthosis as well as durability. As the system is used by people who suffer from obesity, lightness is always an extremely important factor.

The orthosis has one DC motor as an actuator-located hip zone. The actuator system of AHKAFO has two mechanical structures. The first is a gear and T-type deflector reducer mechanism used to transmit the generated torques of the actuator to the hip joints. The other is a pulley and four-bar mechanism, which is used for transferring the generated torque to the knee joints. Thanks to this mechanism the system takes action from the hip zone and performs only the "flexion" and "extension" motions.

Before production, kinematic analysis of the A-HKAFO was carried out using the SolidWorks Motion program to determine the torque and power requirements. During the analysis of the system, the walking cycle of the orthoses device was completed in 2.01 seconds. The necessary torque, power, and angular displacement values for the hip joint were obtained from the analysis results of the device, which carries a maximum mass of $70 \mathrm{~kg}$. 
Figure 2: A-HKAFO device

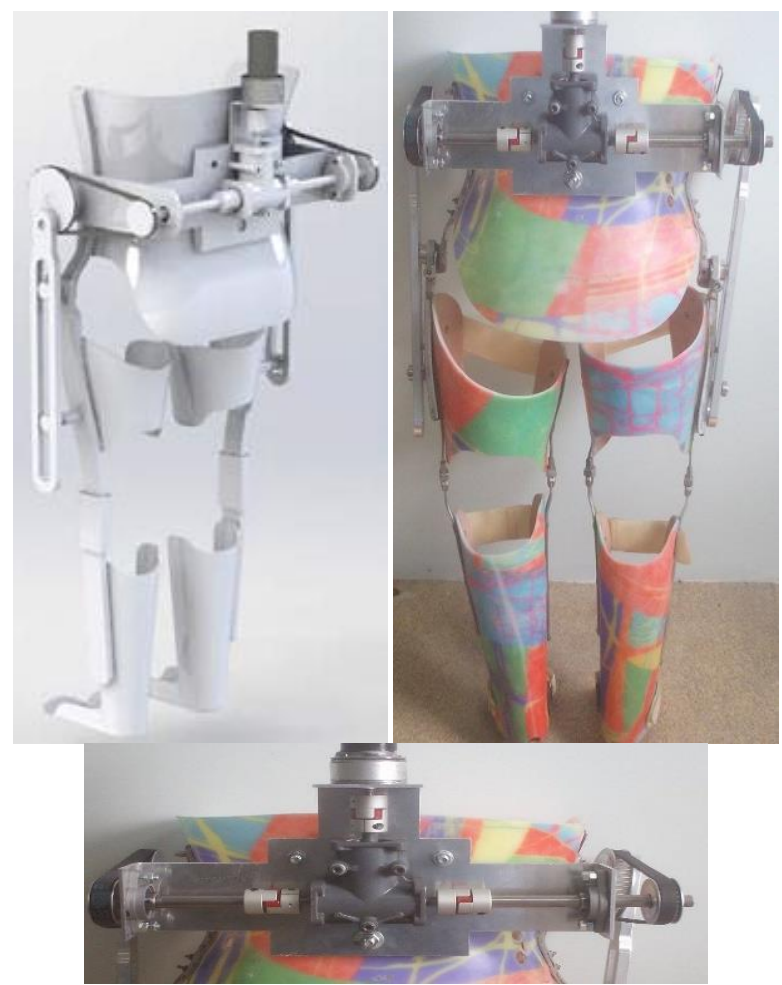

As shown in Figure 3, the maximum torque is approximately $60 \mathrm{Nm}$ for the hip joint. The maximum torque was realized during the stance phase, as expected. If we look at the power value, an average of $100 \mathrm{~W}$ of power is needed. for the orthosis. Also, the angular displacement is shown in Figure 3.

Figure 3: Torque, power, and angle displacement values of the hip joint

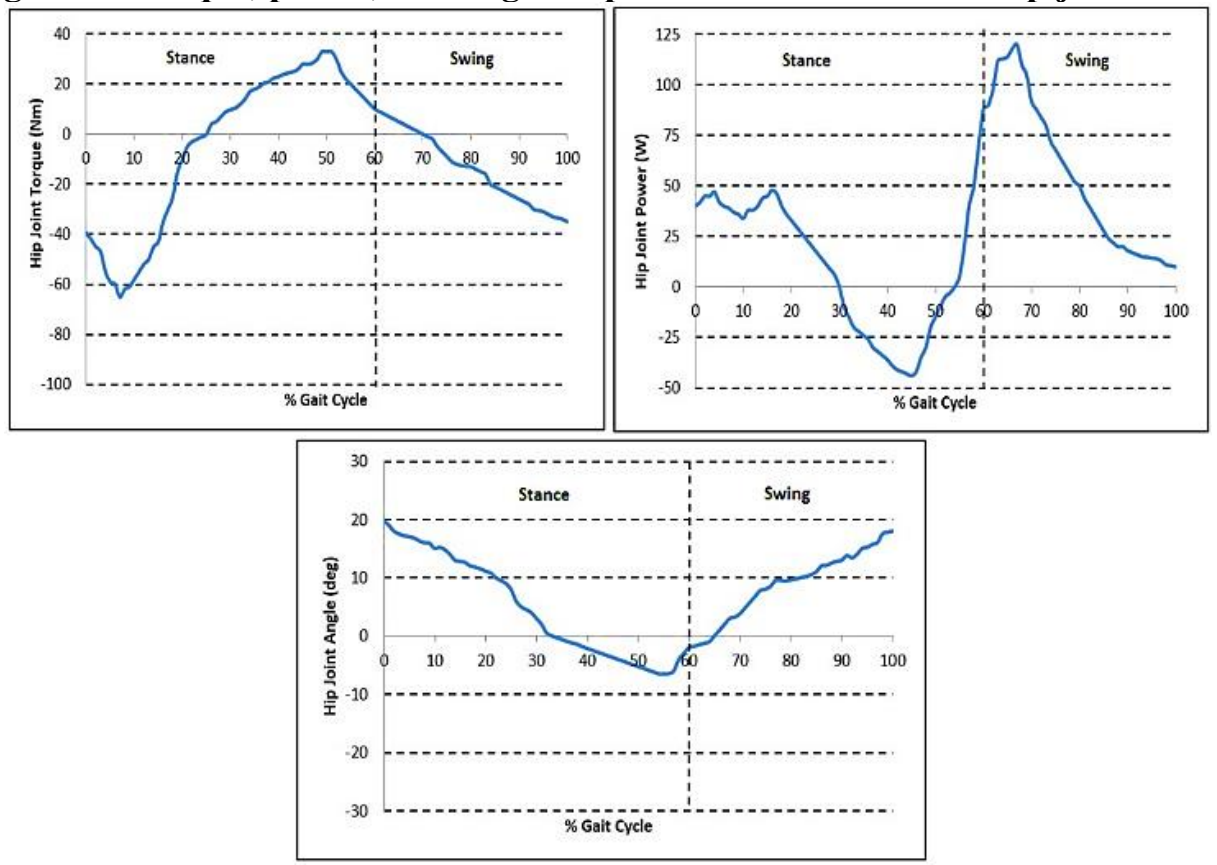


The relation between two different types of torques was determined for modelling the electrical motor used in the A-HKAFO device. The first torque is the required torque of the A-HKAFO joint $T(j)$ to realize determined walking movements and the second torque is the necessary torque that must be supplied by the motor $T(m)$.

The reducer in the high cycle rate $(n)$ must be added to the motor, as the obtained output torque from the electrical motor is much smaller than the necessary joint torques. Because of this, the motor angle $\theta_{m}$, the velocity $\dot{\theta}_{m}$ and the acceleration $\ddot{\theta}_{m}$ are changed to the A-HKAFO joint angle $\theta_{j}$, velocity $\dot{\theta}_{j}$, and the acceleration $\ddot{\theta}_{j}$ according to cycle rate $(n)$. These can be expressed in the equations below:

$$
\begin{aligned}
& \theta_{j}=\frac{\theta_{m}}{n} \\
& \dot{\theta}_{j}=\frac{\dot{\theta}_{m}}{n} \\
& \ddot{\theta}_{j}=\frac{\ddot{\theta}_{m}}{n}
\end{aligned}
$$

(4):

The relation between the motor torque and the A-HKAFO joint torque is shown in equation

$$
T(m)=\frac{T(j)}{n}
$$

The generated torque by the motor must counterbalance the inertia of the reducer. Also, the efficiency of the reducer $\eta(r e d)$ and the expected friction losses $(F)$ must be added to the model of the motor. The new torque equation for the motors is:

$$
T(m)=\frac{T(j)}{n . \eta(\text { red })}+I(\text { red }) \cdot \ddot{\theta}(\text { red })+F
$$

The torque values required for the electric motor in the A-HKAFO device are calculated using Equation 5. For determining of the required torque $T(j)$ for the joints in equation (5), clinical gait data analysis was used. For the selection of the electric motor, the torque-velocity curves obtained by using equation (5) must be compared to the limit curves of the selected motor. The motor is suitable if the motor torque-velocity curves are under the values of motor limits. In this study, the Maxon RE-35 DC motor has been chosen as an actuator. This motor has suitable specifications as it has small dimensions, is lightweight, and has high efficiency. The properties of active orthosis device are given in Table 1.

Table 1. Properties of active orthosis device

\begin{tabular}{cccc}
\hline Orthosis & Weight $(\mathbf{k g})$ & Length $(\mathbf{m m})$ & Material \\
\hline \multirow{2}{*}{ Actuator } & 9.5 & $650 \pm 80$ & Thermoplastic and aluminum \\
\hline & Weight $(\mathbf{k g})$ & Power Rating $(\mathbf{W})$ & Gear Reduction \\
\hline & 0.8 & 90 & $1: 64$ \\
\hline
\end{tabular}

The orthosis has one actuator and the total electrical power is of $90 \mathrm{~W}$. The electrical circuit of the A-HKAFO includes a DC motor; a Microcontroller board; EMG sensors; an accelerometer and a battery. The actuator, gears, and deflector reducer were chosen due to its lightweight features and suitability.

The results of the required torques and powers based on experiments on an 80-kg. -human, with a height of $180 \mathrm{~cm}$, are shown in Table 2 (Crowell et al., 2002). Clinical gait analysis (CGA) has been referenced so that it allows for gathering information about joint kinematics and kinetics of the musculoskeletal system during walking. It has been determined that the active orthosis provides the needed torque and power values, according to the kinematic analysis results from this designed device. 
Table 2. Torque and power values

\begin{tabular}{cccc}
\hline & Hip Extension & Knee Extension & Ankle Extension \\
\hline No-Load Torque (N.m) & 61 & 48 & 118 \\
\hline Load Carrying Torque (N.m) & 88 & 69 & 170 \\
\hline No-Load Power (W) & 110 & 86 & 212 \\
\hline Load Carrying Power (W) & 158 & 140 & 305 \\
\hline
\end{tabular}

\section{CONTROL SYSTEM STRCUTURE OF THE A-HKAFO}

The main control system block diagram of the A-HKAFO is shown in Figure 4. The patient's intention to perform a flexion or extension motion is detected by both EMG and the the accelerometer sensors. To determine the last location of the patient after movement, physical feedback is utilized from the mechanical system.

Figure 4: Control block diagram of the A-HKAFO

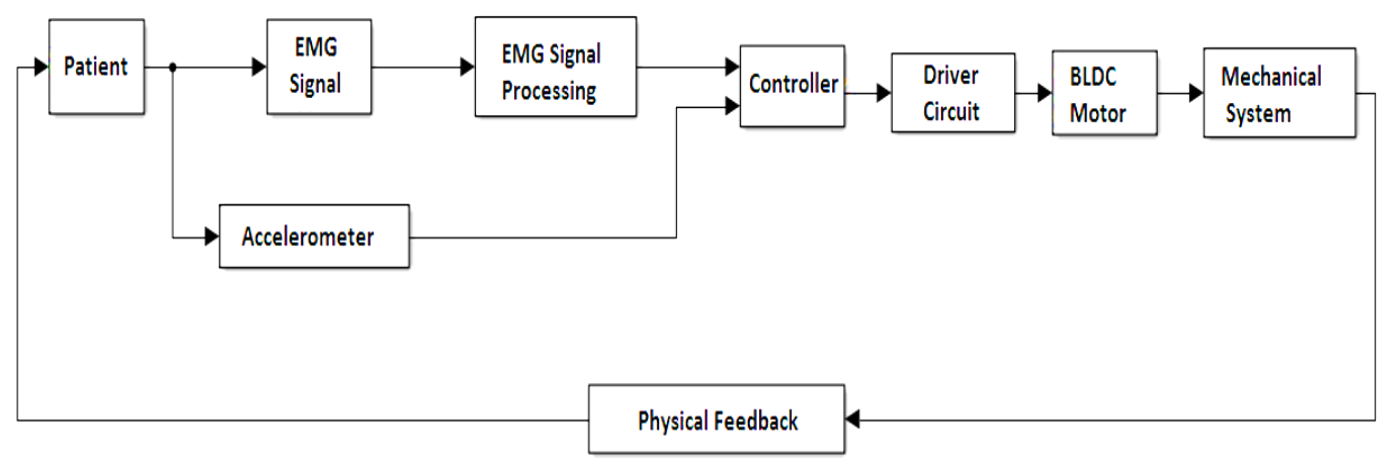

The programming card based on the Microchip ATmega328P has been chosen as controller of the assistive system. It detects walking movements through sensors. During walking, two accelerometers and the surface EMG electrodes have been used for the detection of motion data. An H-bridge motor driver has been used for driving the motor that enables the activation of the assistive system. A tripleaxis digital accelerometer (ADXL345) has been used in this study. The accelerometer sensor is placed on the semitendinosus muscle in the thigh region, allowing three-axis outputs. Only the sensor placed in the $\mathrm{X}$ direction will determine which leg moves. It is a small, thin, low power with high resolution (13 bit) measurement at up to +/-16 g. The illustration of the control circuit is shown in Figure 5. 
Figure 5: Illustration of the control circuit

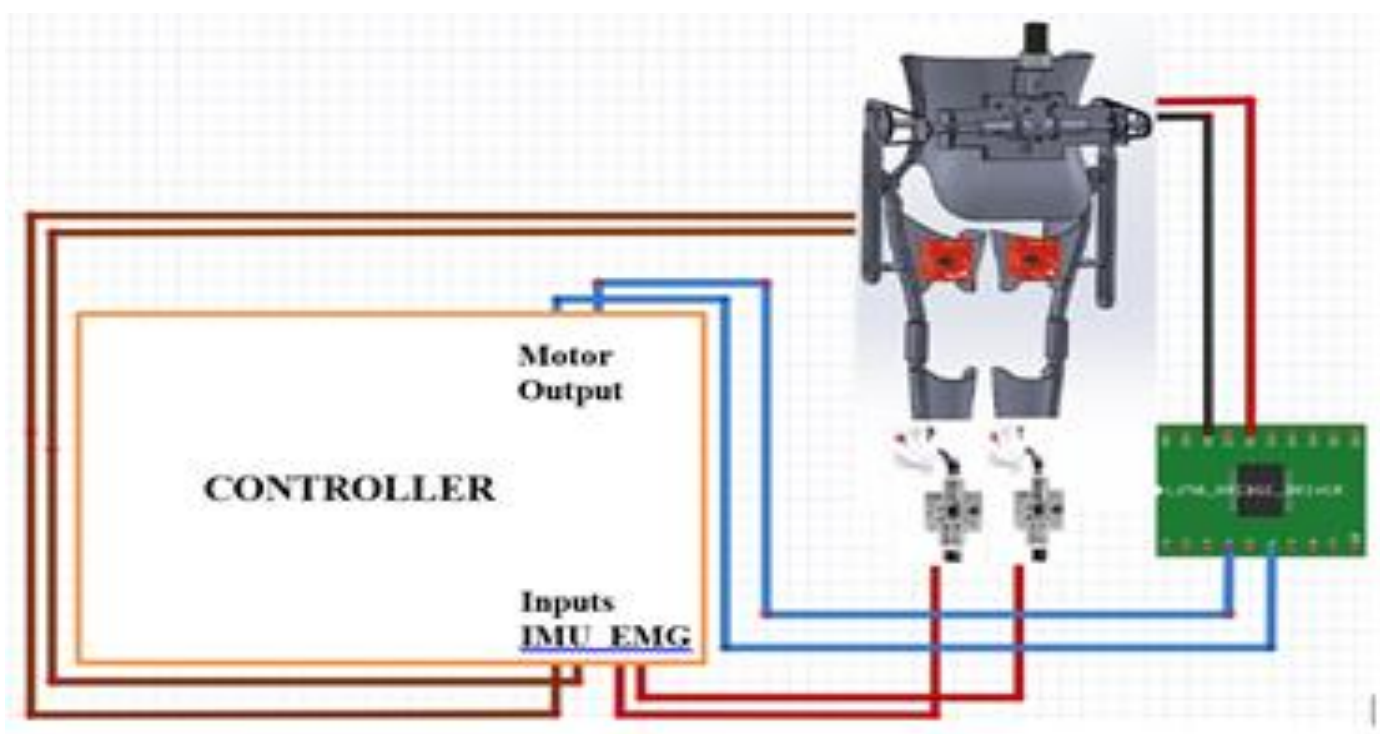

The surface myoelectric signal has been used many important applications in research and clinical practices. The analyses of the data collected through EMG signals consists of several stages: data collection, feature extraction, classification algorithm, and estimation of misclassification (Phinyomark et al., 2012). All of the approaches to EMG pattern recognition have the fundamental processing stages, as shown in Figure 6.

Figure 6. Stages of signal processing for EMG

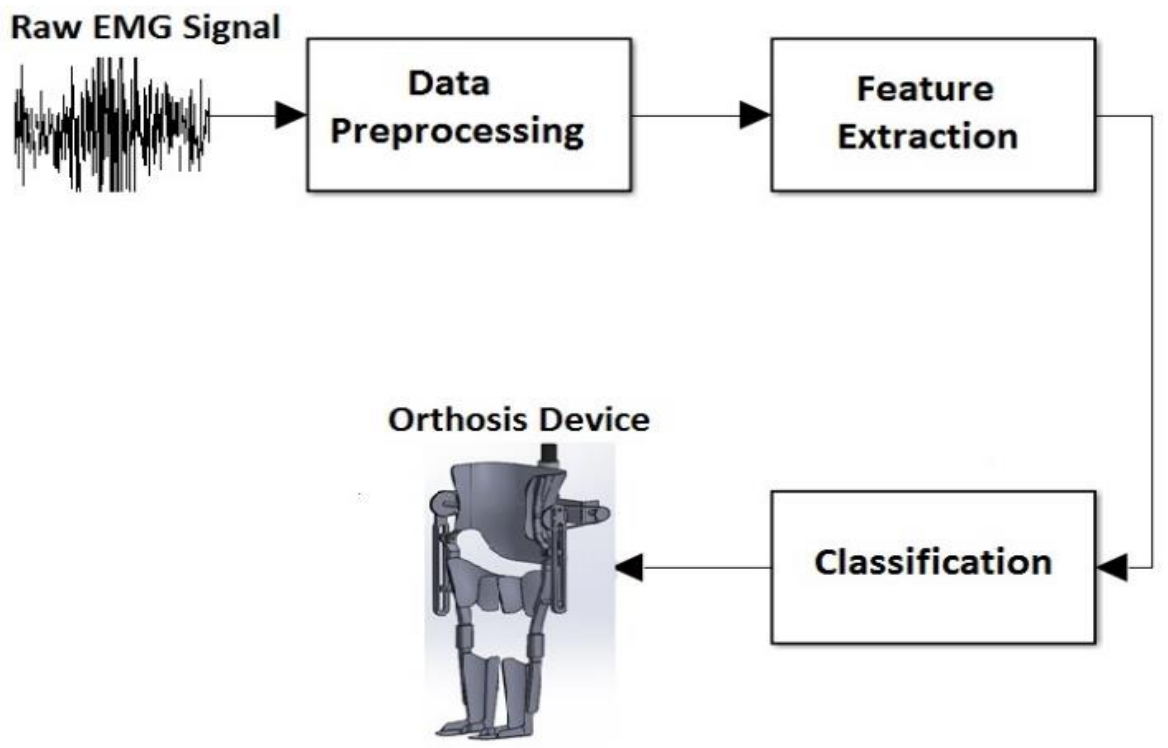

The EMG signals may be subject to pre-processing to remove unwanted interference; the most common sources of interferences are power line harmonics and motion artefact from electrode movement. Since myoelectric signals have a time sequence with a random number of elements, it is not practical for classification. Therefore, the signal sequence should be mapped to feature vectors. Feature vectors of EMG signals are classified to detect which movement produces specific results. For this classification, 
methods like artificial neural networks, fuzzy logic, finite state machine, and support vector machine, etc., classifiers were used (Young et al., 2013; Altan et al., 2019).

In this study, the Finite State Machine (FSM) was chosen as a classifier. The FSM consists of a status set, input, output, event set, and state transition functions. The behavior of each system's state is characterized by a possible system state. Here, the transitions between output states are provided, depending on the input variable and the present state of the system.

\section{EMG BASED CONTROLLER}

A raw EMG signal measured from the gastrocnemius muscle is given in Figure 7. These signals have been obtained from the EMG sensors, attached to the muscle, using surface electrodes. In this study, a single channel has been enough for receiving a single intentional data. The signal is filtered using high pass/low pass filters for noise reduction, and the absolute value of EMG signals is calculated and normalized between $0-1$. The maximum and minimum values of the signals as well as Equation 6 are used for normalization. The filters applied to achieve this signal in the desired frequency range (50-500 $\mathrm{Hz}$ ) are designed as digital filters via the controller. The available EMG signals are predominantly within this frequency range mentioned above.

$$
E M G(t)=\left(E M G_{\text {ham }}(t)-E M G_{\min }(t)\right) *\left(\frac{1}{E M G_{\max }-E M G_{\min }}\right)
$$

Figure 7. Raw EMG signal

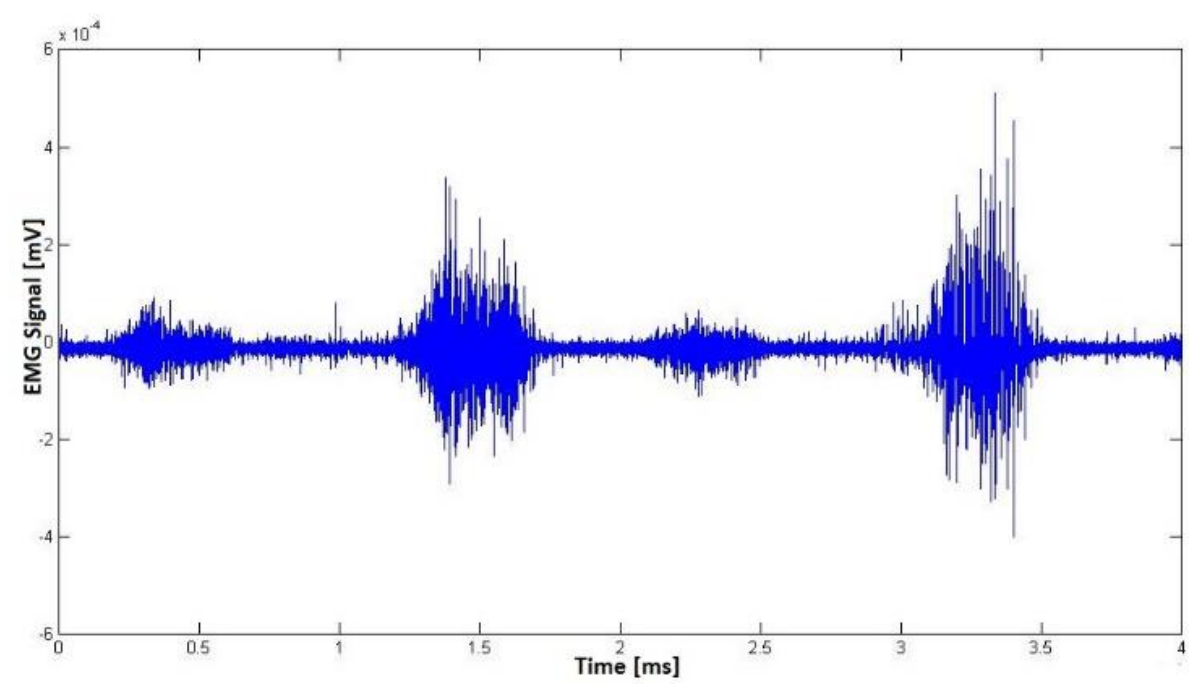

The filtered and normalized EMG signal is shown in Figure 8. A threshold value is defined for converting EMG signals to logical signals and for using the signals for control systems. A logical value 1 is produced when the the muscle contractions exceed this threshold, and a logical value 0 is produced when the muscle contractions fall below this threshold. The patient's physician determines the threshold levels. The threshold value is 0.18 for this patient. After this process, the classification process starts. The signals to be used in the Finite State Machine are also given in Figure 8. 
Figure 8. Muscle contraction conditions
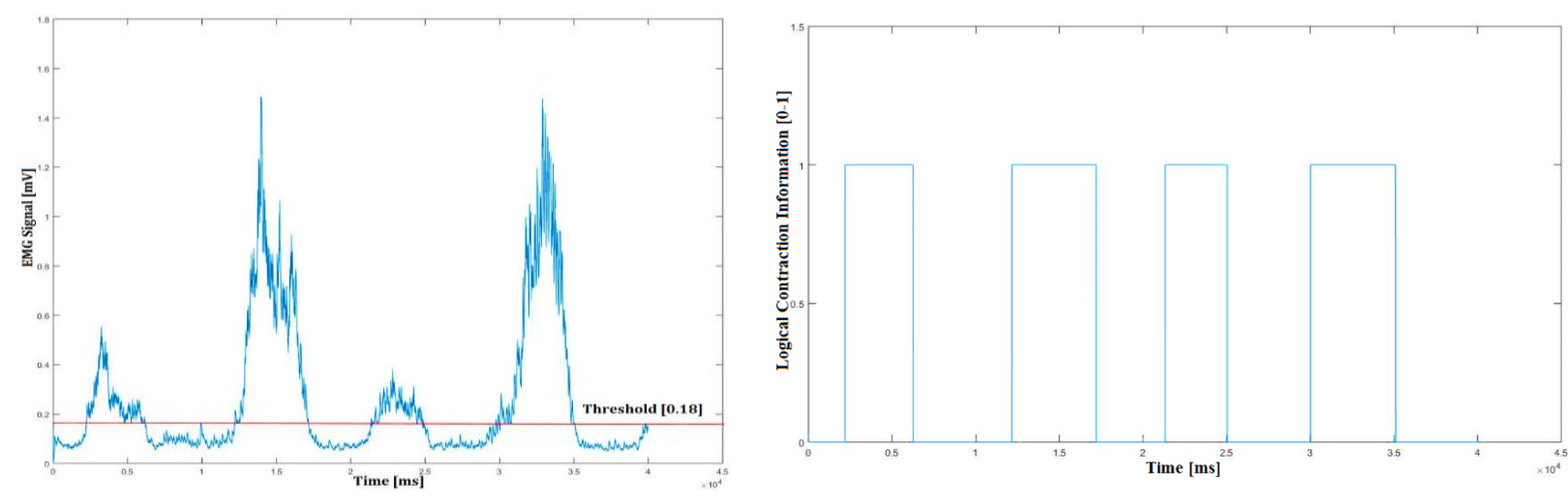

The EMG signals and the accelerometer data collected from both legs are classified using the FSM method, as shown in Figure 9. The result of this classification is used for three different situations of the actuator input. These situations are: the patient stops, moves his/her right leg or moves his/her left leg, respectively. In Figure 9, EMG_Right shows EMG signal measured from the right leg, EMG_Left shows EMG signal measured from the left leg, Adxl_Right shows the position information measured from the right leg and Adxl_Left shows the position information measured from the left leg. The classification has been performed according to the state of these data. The signals sent to the motor driver are expressed as "a" (logic 1 or 0$)$ and "b" (logic 1 or 0$)$.

\section{CONCLUSION}

This paper discusses how an A-HKAFO device, powered by a DC motor, is designed and tested on a person. The device assists people who encounter difficulties in walking due to reasons such as an injured spinal cord, sports injuries, or obesity.

The A-HKAFO, which has been designed by the basic mechanics of human walking, ensures active walking operations for the user with the help of a support system located in the hip zone

A control method has been applied using the Finite State Machine method, based on the acquired EMG signals and the kinematics data taken from accelerometers. This method aims to identify the region from where motion starts by comparing two signals received from the system. A motor located in an AHKAFO will start to work when the person starts moving, and will also ensure that the leg moves forward for the next step. One of the aims of the study is to determine if battery usage could be reduced. This has been achieved through a mechanical system built for actuator-enabled movement (in both directions), and through power reduction.

One of the important design criteria for such systems is the choice of the actuator with the desired weight and size which can be suitable for the system in terms of controllability and low power efficiency. In this study, the selected DC motor has met the desired criteria in terms of weight/performance ratio.

Although the device successfully tested on two patients at Marmara University orthopedic department, a more in-depth trial is needed for its clinical approval. The orthotic devices are usually specially designed for personal use. With its mechanical structure, the developed A-HKAFO device can be used by people of different sizes As a result of some anatomically limited restrictions on the device, providing this support may have some disadvantage. It is aimed to obtain a more appropriate structure for human anatomy by removing these restrictions in the future. It is also aimed to develop a haptic structure so that the system will be smarter by carrying out studies on adapting to the patient's contact sensation. 
Figure 9. The classification of the processed signal using the FSM algorithm

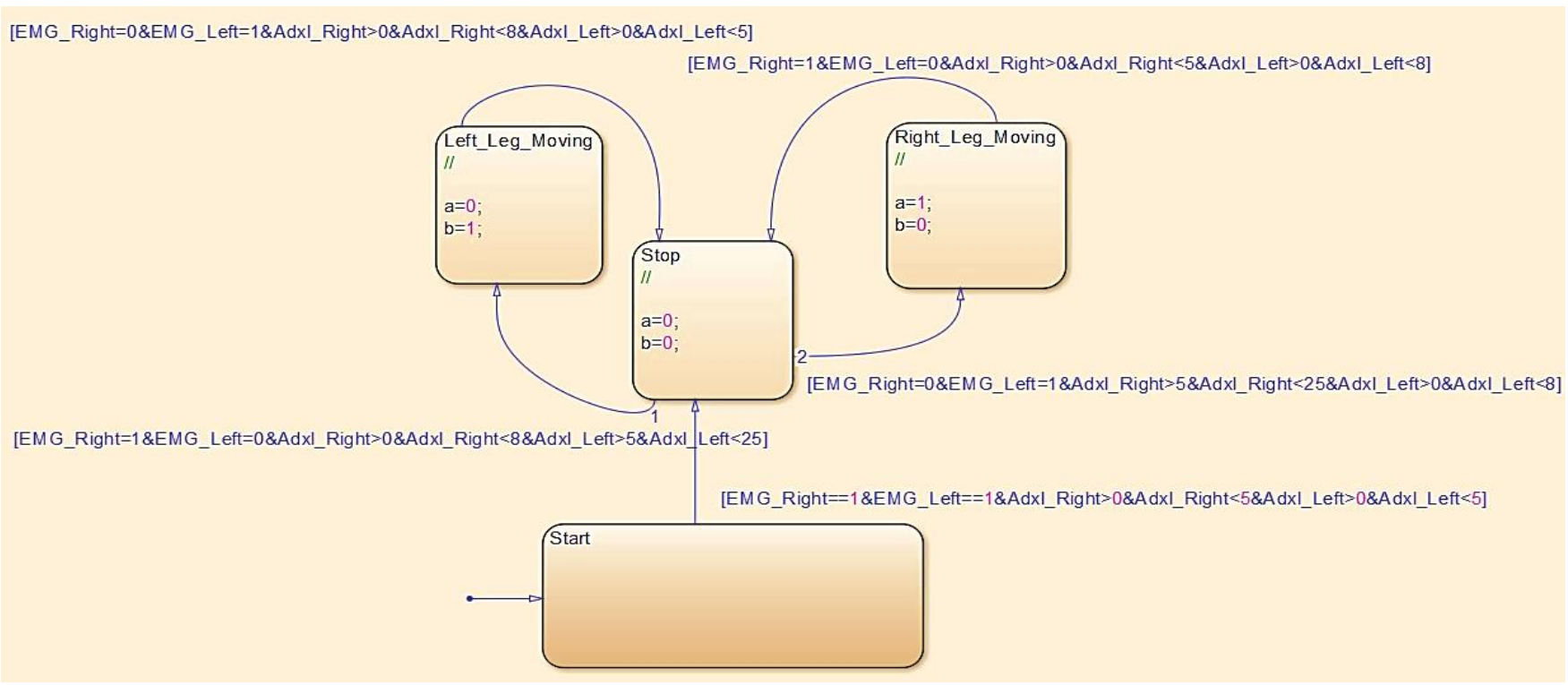

\section{REFERENCES}

[1] Costa, N., Darwin, G.C. (2006). Control of a biomimetic soft-actuated 10 dof lower body exoskeleton, International Conference on Biomedical Robotics and Biomechatronics, 495501.

[2] Hawran, S., Fin, B.S. (1996). The use of long leg calipers for paraplegic patients: a follow-up study of patients discharged, Spinal Cord, 34(11): 666-668.

[3] Lum, P.S., Charles, G.B., Peggy, C.S., Matra, M., Machiel, V.L. (2002). Robot-Assisted movement training compared with conventional therapy techniques for the rehabilitation of upper-limb motor function after stroke, Physical Medicine and Rehabilitation, 83(7): 952-959.

[4] Nagasaka, K., Hirochika, I., Masayuki, I. (1999). Dynamic walking pattern generation for a humanoid robot based on optimal gradient method. IEEE SMC'99 Conference Proceedings, 908-913.

[5] Dollar, A.M., Hugh, H. (2008). Lower extremity exoskeletons and active orthoses: challenges and state-of-the-art, IEEE Transactions on Robotics, 24(1): 144-158.

[6] Giovacchini, F., Federica, V., Matteo, F., Marco, C., Mario, C., Andrea, P., Tingfang, Y., Dirk, L., Nicola, V. (2015). A Light-weight active orthosis for hip movement assistance, Robotics and Autonomous Systems, 73(5): 123-134.

[7] Tucker, M.R., Jeremy, O., Anna, P., Hannes, B., Mohamed, B., Olivier, L., Jose, R.M., Robert, R., Heike, V., Roger, G. (2015). Control strategies for active lower extremity prosthetics and orthotics: a review, Journal of Neuroengineering and Rehabilitation, 12(1): 110-117.

[8] Ulkir, O., Akgun, G., Kaplanoglu, E. (2018). Mechanical design and analysis of a pneumatic ankle foot orthosis. In 2018 Electric Electronics, Computer Science, Biomedical Engineerings' Meeting (EBBT) (pp. 1-4). IEEE.

[9] Shortar, A., Nazha, H., Sliman, A., \& Abbas, W. (2020). Ankle-foot orthosis design between the tradition and the computerized perspectives. The International Journal of Artificial Organs, 43(5), 354-361.

[10] Yan, T., Marco, C., Calogero, M.O., Nicola, V. (2015). Review of assistive strategies in powered lower-limb orthoses and exoskeletons, Robotics and Autonomous Systems, 64(8): 120-136.

[11] Shorter, K.A., Jicheng, X., Elizabeth, H.W., William, K.D., Geza, F.K. (2013). Technologies for 
powered ankle-foot orthotic systems: possibilities and challenges, IEEE/ASME Transactions on Mechatronics, 18(1): 337-347.

[12] Dollar, A.M., Hugh, H. (2007). Active orthoses for the lower-limbs: challenges and state-of-theart, IEEE 10th International Conference on Rehabilitation Robotics, 968-977.

[13] Samuel, A., Berniker, M., Hugh, H. (2008). Powered an-kle-foot prosthesis to assist level-ground and stair-descent gaits, Neural Networks, 21(4): 654-666.

[14] Shorter, K.A., Kogler, G.F., Loth, E., Durfee, W.K., Hsiao-Wecksler, E.T. (2011). A portable powered ankle-foot orthosis for rehabilitation, Journal of Rehabilitation Research \& Development, 48(4): 459-472.

[15] Sawicki, G.S., Gordon, K.E., Ferris, D.P. (2005). Powered lower limb orthoses: applications in motor adaptation and rehabilitation, 9th International Conference on Rehabilitation Robotics, 206-211.

[16] Ferris, D.P., Gordon, K.E., Sawicki, G.S., Peethambaran A. (2006). An improved powered anklefoot orthosis using proportional myoelectric control, Gait \& Posture, 23(4): 425-428.

[17] Kanthi, M., George, V.I., Mruthyunjaya, H.S. (2014). Embedded system design to control active ankle foot orthosis, Journal of Consumer Electronic Times, 3(1): 206-214.

[18] Crowell, Harrison, P., Angela, C.B., Michael, M. (2002). Exoskeleton power and torque requirements based on human biomechanics, Army Research Lab Aberdeen Proving Ground $M D$.

[19] Phinyomark, A., Phukpattaranont, P., Limsakul, C. (2012). Feature reduction and selection for EMG signal classification, Expert Systems with Applications, 39(8): 7420-7431.

[20] Young, A.J., Smith, L.H., Rouse, E.J., Hargrove, L.J. (2013). Classification of simultaneous movements using surface EMG pattern recognition. IEEE Transactions on Biomedical Engineering, 60(5), pp.1250-1258.

[21] Altan E., Pehlivan K, and Kaplanoğlu E, (2019), Comparison of EMG Based Finger Motion Classification Algorithms, 27th Signal Processing and Communications Applications Conference (SIU), Sivas, Turkey, 2019, pp. 1-4, doi: 10.1109/SIU.2019.8806331. 